\title{
LINE BY TESTER ANALYSIS FOR YIELD AND YIELD COMPONENTS IN HYBRID RICE \\ EL-Diasty, Z.M. ${ }^{1}$; H.F. EL-Mowafi ${ }^{2}$; M.S. Hamada ${ }^{3}$ and \\ E. M. Belih ${ }^{1,2}$ \\ 1- Genetics Dept., faculty of Agric., Mansoura University, Egypt. \\ 2- Rice Res. And Training Center (RRTC), Sakha, Kafr EL-Sheikh, Egypt. \\ 3-Genetics Dept., faculty of Agric., Damietta Branch, Mansoura University, Egypt.
}

\begin{abstract}
This study was conducted to investigate combining ability and gene action of rice hybrids and their parental lines. Four CMS lines as female parents and three restorers' lines as male parents were hybridized to produce $12 F_{1}$ hybrids were used in a line $x$ tester mating design. the resulted genotypes were used to estimate combining ability, heterosis, and gene action for yield and yield components characters. The results indicated that most characters were largely governed by additive gene action. General and specific combining ability effects of the genotypes for the characters under study were estimated. The results showed that the characters were controlled both general combining ability (GCA) and specific combining ability (SCA). The CMS and restorer lines showed variable effects for GCA. The study also indicated the presence of heterosis over the better parent (BP), Mid-parents (MP) and over the best check variety Giza 178 for nine characters of yield and yield components.
\end{abstract}

\section{INTRODUCTION}

Rice has become one of the main factors of food security for the world population. Hybrid rice offers from $15-20 \%$ yield advantage over the high-yielding inbred varieties under similar cultivation conditions. In order to obtain good hybrids, it is essential to understand the nature of gene action that controlls yield and its components. Combining ability estimation is a powerful tool available to select the desirable parents and crosses for the exploitation of heterosis. General combining ability (GCA) effects largely involves additive gene effects, whereas specific combining ability (SCA) effects represent only non-additive gene action including dominance. The presence of non-additive genetic variance offers scope for exploration of heterosis (Yadav et al., 1999). The parents with good GCA and SCA could good hybrids (Yan et al., 2000). The rice researchers around the world carried out many studies on combining ability of different lines of rice among them, (Sun et al., 1993; Gong et al., 1993; Chen et al., 1997).Their achievements in this aspect Could recommend the use of hybrid rice by selecting good parents in hybridization breeding program. In addition, heterosis and combining ability of hybrid rice were studied by Zhang et al., (2002). Line $x$ tester analysis provides information about GCA and SCA effects of parents and is helpful in estimating various types of gene actions (Rashid et al., 2007). Identification of suitable parents through line $x$ tester analysis in rice has been studied by Singh \& Kumar (2004). 


\section{EL-Diasty, Z.M.et al.}

The present study included the utilization of four CMS lines $x$ three testers to produce $12 \mathrm{~F} 1 \mathrm{~s}$. All the hybrids and the seven rice genotypes were used to estimate combining ability, heterosis, as well as gene action for yield and yield components.

\section{MATERIALS AND METHODS}

Four cytopasmic male sterility lines (CMS) vis., IR68885A mutant type, IR69625A possessing wild abortive type (WA) ,G46A possessing Gambiaca type and Large stigma A possessing Kalinga type of cytoplasm, were used as female lines (Table 1). These CMS lines were crossed with three elite lines/varieties Giza 178R, Giza 182R and GZ5121-5-2R to generate 12 hybrid combinations in line $\mathrm{x}$ tester mating design. The hybrids were evaluated along with parents in a randomized complete block design with three replications at Rice Researsh Training Center during 2006 season. Thirty-day-old seedlings were transplanted ( Each plot was made of seven rows $5 \mathrm{~m}$ long and spaced at $20 \mathrm{~cm}$ apart, $20 \mathrm{~cm}$ interplant distance). Days to flowering, plant height, panicle length, panicle weight, filled grains panicle ${ }^{-1}$, spikelets panicle ${ }^{-1}$, spikelet fertility percentage, 1000-grain weight and grain yield tha ${ }^{-1}$ were noted. Combining ability analysis was carried out following the method of kempthorne (1957).

Table (1): Cytopasmic male sterile lines and restorer lines used for the study.

\begin{tabular}{|l|c|c|}
\hline Genotypes & Cytoplasm source & Origin \\
\hline CMS lines (female) & Mutant IR62829A & IRRI \\
\hline IR68885A & WA & IRRI \\
IR69625A & Gambiaca & China \\
G46A & Kalinga & China \\
Large stigma A & & \\
Restorer lines (male) & Restorer & Egypt \\
\hline Giza 178R & Restorer & Egypt \\
Giza 182R & Restorer & Egypt \\
GZ5121-5-2R & Rer \\
\hline
\end{tabular}

\section{RESULTS AND DISCUSSION}

The analysis of variance revealed that the variation in genotypes (parents and hybrids) was highly significant for all the studied characters (Table 2). The parents, hybrids and interaction between parents and hybrids showed highly significant differences for all characters. The analysis of variance for combining ability (Table 2) also showed highly significant differences among the lines for all the characters. While the testers exhibited highly significant differences for all the characters except for spikelets panicle1 , which was non-significant. The significance of the mean of sum squares due to lines and testers indicated a prevalence of additive variance. However, means of sum of squares due to line $x$ testers were also significant for all the characters except for 1000-grain weight, which indicating that the importance of both additive and non-additive variance. Several workers reported the predominance of dominant gene action for a majority of the yield trails (Ramaligan et al., 1993; Peng and Virmani 1990 and Stayanarayana et al., 
1998). Also EL-Mowafi 2001a, EL-Mowafi and AbouShoush (2003), Singh and Kumar (2004), EL-Mowafi et al., (2005), Abd EL-Hadi and EL-Mowafi (2005) and EL-Diasty et al., (2008) have reported the same results.

\section{Genetic Parameters:}

The estimates of genetic parameters viz, additive variance $\left(\sigma^{2} A\right)$, dominance variance $\left(\sigma^{2} D\right)$, environmental variance $\left(\sigma^{2} E\right)$, genotypic variance $\left(\sigma^{2} G\right)$, and phenotypic variance $\left(\sigma^{2} P\right)$, broad sense heritability $\left(h^{2} b\right)$, narrow sense heritability $\left(h^{2} n\right)$, relative importance of GCA \% and relative importance of SCA \% for all nine studied characters are presented in Table (3). It is revealed that the estimation of additive genetic variance $\left(\sigma^{2} A\right)$ and relative importance of GCA \% for all studied characters except for grain yield were higher than non-additive or dominance genetic variance $\left(\sigma^{2} D\right)$ and relative importance of SCA \% for these characters. These results indicated that the former characters were largely governed by additive gene action. The importance of additive gene action for the inheritance of these characters was supported with similar results obtained by Lokaprakash et al. (1991), ELMowafi (1994), Sharma and Koranne (1995), EL-Mowafi (2001a), Attia. (2001), EL-Refaee (2002), EL-Mowafi and Abou shousha (2003), Ahmed (2004), EL-Mowafi et al. (2005), Abd EL-Hadi and EL-Mowafi (2005) and ELDiasty et al. (2008). On the other hand, high estimate of dominance genetic variance and its relative magnitude of SCA \% were found to be more than that of additive variance for grain yield characters. This result indicated that dominance genetic variance played a major role in the inheritance of this character, which was in general agreement with the results reported by Sharma and Koranne (1995), Ramalingam et al. (1997) and EL-Refaee (2002), and EL-Mowafi and Abou-Shousha (2003). Heritability estimation in broad sense was high for all characters under study as given in Table (3). Heritability estimates were high for plant height, panicle length, filled grains panicle $^{-1}$, spikelets panicle ${ }^{-1}$, spikelet fertility $\%$ and 1000-grain weight and moderate for heading date and panicle weight, indicating that a major part of the total genotypic is additive, while heritability estimation in the narrow sense for grain yield tha-1 was low.

\section{Estimation of General Combining Ability (GCA) Effects:}

The estimation of the general combining ability (GCA) effects of lines and testers revealed that the parents: IR68885A and IR69625A, Giza 178R and Giza 182R were good combiners for earliness and dwarfness (Tables 4 and 5). While, IR68885A, Giza 182R were found to be better combiners for panicle length. Whereas, G46A, IR69625A, Giza 182R and Giza 178R appeared to be better combiner for panicle weight. Large stigma, G46A, Giza 178R, and Giza 182R were good for filled grains panicle ${ }^{-1}$; and spikelets panicle $^{-1}$. The lines : IR69625A, Giza 182R and Giza 178R had high GCA effects for spikelet fertility \%; Large stigma A. Also, the lines : G46A, Giza $182 R$ and Giza 178 were found to be better combiners for 1000-grain weight. While, IR69625A, G46A, and Giza 178R were identified as better combiners for grain yield. 
EL-Diasty, Z.M.et al.

2-3-4

8508 


\section{Estimation of Specific Combining Ability (SCA) Effects:}

The usefulness of a particular cross in exploiting heterosis is judged by the specific combining ability (SCA) effect. The estimation of SCA of 12 crosses for nine characters are presented in Table 6 . It is observed that out of 12 hybrid combinations, there were three hybrids exhibited positive and significant SCA for grain yield. The promising combinations for grain yield along with other characters were: IR68885A/GZ5121R, IR69625A/GZ5121R and G46A/Giza 182R. It was observed that the majority of these crosses had high SCA for grain yield involved with higher/low or average/low general combining parents, while there was one hybrid combination showed low/low general combiners with high SCA. The hybrid combinations that showed high negative SCA for days to heading (earliness) were : IR69625A/GZ5121R, Large stigma A/Giza 182R, G46A/Giza 182R and IR68885R/Giza 178R. For plant height, estimates of SCA were obtained where among these hybrids desirable and the good specific combiners for this were: G46A/GZ5121R, IR69625A/Giza 182R, Large-stigma A/GZ5121R, IR68885A/Giza 178R, and IR68885A/Giza 182R. The hybrid combinations, G46A/GZ5121R, IR69625A/Giza 182R/Giza 182R and large-stigma A/Giza 178R were good combiners for panicle length. The best specific combiners for panicle weight were G46A/Giza 178R, IR69625A/ GZ5121R, IR68885A/Giza 182R, largestigma A/Giza 178R, and IR68885A/ GZ5121R. The hybrid combinations, IR68885A/ GZ5121R and IR69625A/Giza 182R showed higher SCA for filled grains panicl ${ }^{-1}$ and spikelets panicle ${ }^{-1}$. For spikelet fertility \%, the hybrid of IR68885A/ GZ5121R, IR69625A/Giza 182R, G46A/Giza 178R, and largestigma A/Giza 178R exhibited high SCA.

\section{Heterosis}

Heterosis percent over better parent (BP), Mid-parents (MP), and over best check variety Giza 178 was calculated for nine yield and yield component characters (Tables 7,8,9). The degree of heterosis varied from hybrid to hybrid, also from character to character. Many scientists have observed varying degrees of heterosis for yield and its related characters (EL-Mowafi et al., 2001 b; Alam et al., 2004,Abd EL-Hadi and El-Mowafi 2005 and EL-Mowafi et al., 2005). For days to heading and plant height, negative heterosis was desirable but for the rest of the characters positive heterosis was desirable. Positive as well as negative $\mathrm{BP}, \mathrm{MP}$, and $\mathrm{SH} \%$ were observed for all the characters (Tables $7,8,9$ ). Some of the hybrids flowered earlier than their early parents. There were also some the hybrids flowered later than late parents. Among the earlier hybrid there was: IR68885A/Giza 178Rwhich exhibited the highest significant heterosis of $-9.00 \%$ over the BP, $-7.40 \%$ over MP and $-9.00 \%$ over standard variety (SH \%) for earliness. Three other hybrids viz. IR69625A/Giza 178R, IR69625A/Giza 182R and G46A/Giza 178R also manifested BP, MP, and SH \% for earliness. Regarding plant height, it was found that the hybrids were mostly tall in height to be classified into either medium tall or tall types. The results indicated that the mean heterosis effects for grain yield were significant and positive in 9 out 12 hybrids, as deviation from the better parent value (Table 7). 
EL-Diasty, Z.M.et al.

5-6-7

8510 
J. Agric. Sci. Mansoura Univ., 33 (12), December, 2008

8-9

8511 


\section{EL-Diasty, Z.M.et al.}

The highest estimates were detected for the hybrids : G46A/Giza 182R (31.89\%), G46A/Giza 178R (27.98\%), IR69625A/Giza178R (26.6) and IR69625A/Giza 182R (21.82\%). Moreover, significant and positive values of heterosis were estimated for 11 hybrids also as deviation from the MP. The highest heterotic effects over MP were $(46.00 \%),(41.81 \%),(31.89 \%)$ and (29.41\%)for hybrids(G46A/Giza 182R, G46A/Giza 178R, Large stigma A/Giza 178R, andIR69625A/Giza178R), respectively. However, out of 12 hybrids, the same nine hybrids exhibited highly significant and positive heterotic effects as deviation from the standard variety (Giza 178R). The highest heterotic effects were $31.63 \%, 27.98 \%, 26.60 \%$ and $21.58 \%$ for the above mentioned hybrids. Significant heterosis for yield and yield characters were also reported by EL-Mowafi (2001 b), EL-Refaee (2002), EL-Mowafi et al., (2005) Rashid et al., (2007) and EL-Diasty et al., (2008).

\section{REFERENCES}

Abd EL-Hadi, A.H. and El-Mowafi (2005) Combining ability analysis of the maintainer and the restorer lines for cytoplasmic male sterile (CMS) system of hybrid rice .Egypt. J. Agric.Res., 83(5A): 183-196.

Ahmed. A. R. M. (2004). Genetically studies on some hybrids of rice. MSc Thesis, Fac. of Agric. Mansoura University, Egypt.

Alam,-M-F; Khan,-- M-R; Nuruzzaman,-M;Paravez,-S; Swaraz,-A-M;Alam,-I and Ahsan,-N(2004).Genetic basis of heterosis and inbreeding depression in rice (Oryza sativa L.).In Journal -of -ZhejiangUniversity-Science. Bangladesh.; 5(4):406-411.

Attia, K.A. (2001). Evaluation and RAPD analysis of photo-thermo-sensitive genetic male sterile lines in Indica rice (Oryza sativa L.). M.Sc. Thesis, Institute of Genetics and Plant Breeding. College of Agric. and Biotechnology, Zhejiang Univ., Hangzhou, P.R. China.

Chen, S.H; H.R. Lu and J.B. Yang (1997). Heterosis combining ability and correlation of agronomic traits in two-line inter-subspecific hybrid rice. Journal Fujian Agric. Univ., 26: 1-7.

EL-Diasty, Z.M.; H. F. EL-Mowafi; M. S. Hamada and R. M. Abdallah. (2008). Genetic studies on the photo/thermo-sensitive genic mail sterility (P/TGMS) and its utilization in rice breeding. Mansoura University Journal.

El-Mowafi, H.F., A.O. Bastawisi, M.I. Abou Youssef and F.U. Zaman (2005). Exploitation of rice heterosis under Egyptian conditions. Egypt. J. Agric. Res. 389 (5A): 143-166.

El-Mowafi, H.F. A.H.Abd El Hadi (2005) Studies on heterosis of some maintainer and restorer lines for cytopasmic male sterile system in hybrid rice. Egyptian J.Agric.Res.,83(5A):169 - 182.

El-Mowafi, H.F. (2001a). Combining ability analysis for some cytopasmic male sterile and restorer rice lines under Egyptian conditions. Egypt. J. Appl. Sci., 16(2): 25-51. 
El-Mowafi, H.F. (2001b). Combining ability analysis for some cytoplasmic male sterile and restorer rice lines under Egyptian conditions. Egypt. J. Appl. Sci., 16(2): 25-51.

El-Mowafi, H.F. and A.A. Abou Shousha (2003). Combining ability and heterosis analysis of diverse CMS lines in hybrid rice. J. Agric. Res. Tanta Univ., 29(1): 106-127.

El-Mowafi, H.F. (1994). Studies on rice breeding. Ph.D. Thesis, Faculty of Agric., Kafr El-Sheikh Tanta University, Egypt.

El Refaee, Y.Z.E. (2002). Genetically and biochemical studies on heterosis and combining ability in rice. M.Sc. Thesis, Fac. of Agric., Tanta Univ., Kafr El-Sheikh, Egypt.

Gong, G.M.; G.F. Zhou, and C.Q. Yin (1993). Analysis on the combining ability of main agronomic traits of Indica double functional genic malesterile lines. Chinese Journal Rice Sci., (7): 137-142.

Kempthorne, O. (1957). An Introduction to Genetic Statistics. John Wiley and Sons Inc., New York, 458-471 pp.

Lokaprakash, R.; G. Shivashankar; M. Mahaderappa; B.T. Shankaregowda and R.S. Kulkami (1991). Combining ability for yield and its components in rice. Oryza 28(3): 319-322 (Cited From Rice Abstracts. 1995 Vol. 18, No. 1)

Peng, J.Y. and S.S. Virmani (1990). Combining ability for yield and four related traits in relation to breeding in rice. Oryza 27: 1-10.

Ramalingam, J.; N. Nadarajan; G. Vanniarajan and P. Rangaswamy (1997). Combining ability studies involving CMS lines in rice. Oryza, 34: 4-7.

Ramalingam, J.; P. Vivekanandan and C. Vanniarajan (1993). Combining ability analysis in lowland early rice. Crop Research-Hisar. 6: 2, 288233; 7 ref.

Rashid M; A.A. Cheema and M. Ashraf. (2007). Line X Tester Analysis in Basmati Rice. Pakistan Journal of Botany, 39(6): 2035-2042.

Satyanarayana, P.V.; I. Kumar and M.S.S. Reddy (1998). Studies on combining ability and heterosis in rice. Int. Rice Res. Notes, 23(3): 10.

Sharma, R.K. and K.D. Koranne (1995). Line $x$ tester analysis for yield and yield components in indica $x$ Japonica crosses of rice. Oryza, 32: 234238.

Singh, N.K. and A. Kumar. (2004). Combining ability analysis to identify suitable parents for heterotic rice hybrid breeding. IRRN, 29(1): 21-22.

Sun, Y,W.; J.Q. Li and Y.B. Liu (1993). The heterosis and combining ability for the days to heading of 'Tow-line system' inter-subspecific hybrid rice. Chinese J. Rice Sci., 7:105-108.

Yadav, L.S.; D.M. Maurya; and S.B. Singh (1999). Combining ability analysis for yield and its components in hybrid rice. Oryza, 36: 208-210.

Yan S.; S. Wen; Z. Li; and C. Liu (2000). Study on the combining ability of Indica two-line hybrid rice. Journal of Huazhong Agric. Univ. 19: 204208.

Zhang R.; J.S. Ming; C.W. Xu; and X.K. Wanc (2002). Heterosis and combining ability of hybrid rice and its relation to Japonica-index of parents. News letters, rice genetics 14: p34. 


\section{EL-Diasty, Z.M.et al.}

تحليل السلالة X الكثاف للمحصول ومكوناته فى الأرز الهجين.

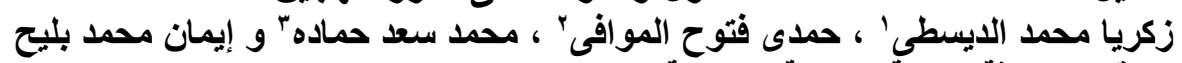

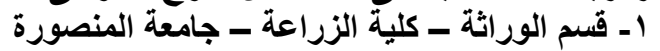

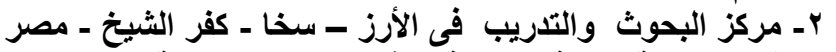

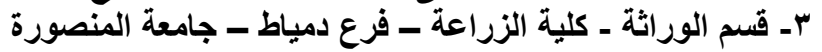

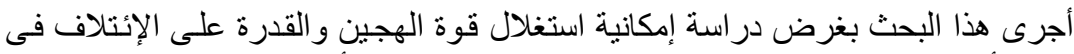

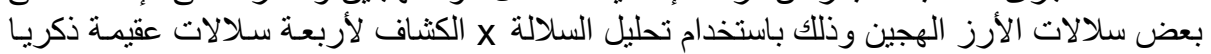

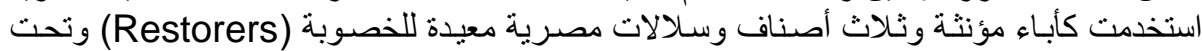

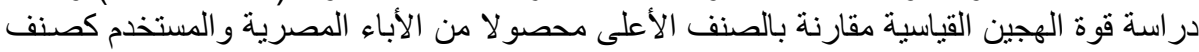

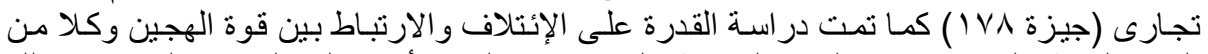

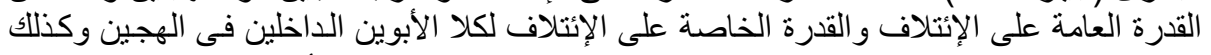

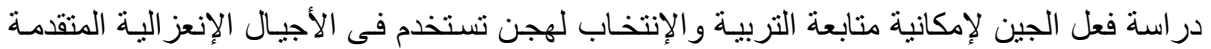

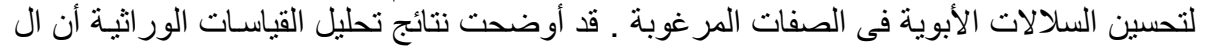

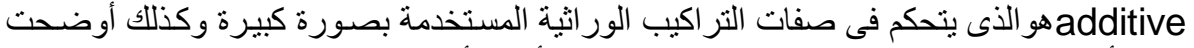
النتائج أن القدرة العامة على الإئتلاف (GCA) كانت أكثر تأثير ا. 
Table (2): Analysis of variance for combining ability.

\begin{tabular}{|c|c|c|c|c|c|c|c|c|c|c|}
\hline Source of variance & d.f & $\begin{array}{c}\text { Heading } \\
\text { date } \\
\text { (days) }\end{array}$ & $\begin{array}{l}\text { Plant height } \\
\text { (cm) }\end{array}$ & $\begin{array}{l}\text { Panicle } \\
\text { length } \\
\text { (cm) }\end{array}$ & $\begin{array}{l}\text { Panicle } \\
\text { weight } \\
\text { (g) }\end{array}$ & $\begin{array}{c}\text { Filled grains } \\
\text { panicle }^{-1}\end{array}$ & $\begin{array}{l}\text { Spikelets } \\
\text { panicle }^{-1}\end{array}$ & $\begin{array}{c}\text { Spikelet } \\
\text { fertility \% }\end{array}$ & $\begin{array}{c}\begin{array}{c}\text { 1000-grain } \\
\text { weight } \\
\text { (g) }\end{array} \\
\end{array}$ & $\begin{array}{c}\text { Grain yield } \\
\text { (t/ha) }\end{array}$ \\
\hline Seplications & 2 & 0.070 & & 0.389 & 0.029 & 34.185 & 20.363 & 2.009 & 0.364 & 0.054 \\
\hline & 18 & & & & & & & & & \\
\hline & 6 & & & & & & & & & \\
\hline & 11 & & & & & & & & & \\
\hline vs hybrids & 1 & & & 90.7 & & & & & & \\
\hline & 3 & & & & & & & & & \\
\hline & 2 & & & & & & & & & \\
\hline Females $\times$ Males $(F \times M)$ & 6 & $56.306^{\star \star}$ & & $0.942^{* *}$ & $0.829^{* *}$ & & & & & $5.379^{* \star}$ \\
\hline rror & 36 & & & & & & & & & \\
\hline $\mathrm{CV} \%$ & & 0.960 & 1.000 & 1.450 & 3.690 & 4.640 & 4.250 & 1.320 & 2.140 & 2.070 \\
\hline
\end{tabular}

*, ${ }^{* *}$ Significant at 0.05 and 0.01 levels, respectively.

Table (3): Genetic parameters for the nine studied characters.

\begin{tabular}{|c|c|c|c|c|c|c|c|c|c|}
\hline parameter & $\begin{array}{l}\text { Heading } \\
\text { date } \\
\text { (days) }\end{array}$ & $\begin{array}{l}\text { Plant } \\
\text { height } \\
(\mathrm{cm})\end{array}$ & $\begin{array}{l}\text { Panicle } \\
\text { length } \\
\text { (cm) }\end{array}$ & $\begin{array}{l}\text { Panicle } \\
\text { weight } \\
\text { (g) }\end{array}$ & $\begin{array}{c}\text { Filled } \\
\text { grains } \\
\text { panicle }{ }^{-1}\end{array}$ & $\begin{array}{l}\text { Spikelets } \\
\text { panicle }^{-1}\end{array}$ & $\begin{array}{c}\text { Spikelet } \\
\text { fertility (\%) }\end{array}$ & $\begin{array}{l}\text { 1000- } \\
\text { grain } \\
\text { weight } \\
\text { (a) }\end{array}$ & $\begin{array}{l}\text { Grain } \\
\text { yield } \\
\text { (t/ha) }\end{array}$ \\
\hline Additive variance $\left(\sigma^{2} \mathrm{~A}\right)$ & 22.910 & 62.800 & 0.675 & 0.293 & 303.240 & 425.990 & 18.240 & 4.680 & 0.272 \\
\hline Dominant variance $\left(\sigma^{2} D\right)$ & 18.450 & 11.380 & 0.272 & 0.268 & 117.310 & 111.200 & 7.820 & 0.067 & 1.780 \\
\hline Environmental variance $\left(\sigma^{2} \mathrm{E}\right)$ & 0.959 & 1.140 & 0.127 & 0.026 & 41.990 & 48.389 & 1.280 & 0.353 & 0.049 \\
\hline Genotypic variance $\left(\sigma^{2} \mathrm{G}\right)$ & 41.360 & 74.180 & 0.947 & 0.561 & 420.550 & 537.190 & 26.060 & 4.750 & 2.052 \\
\hline Phenotypic variance $\left(\sigma^{2} P\right)$ & 42.320 & 75.320 & 1.074 & 0.587 & 462.540 & 585.580 & 27.340 & 5.100 & 2.100 \\
\hline Broad sense heritability $\left(h^{2}\right) \%$ & 0.980 & 0.990 & 0.882 & 0.956 & 0.910 & 0.920 & 0.950 & 0.930 & 0.980 \\
\hline Narrow sense heritability $\left(h^{2}\right) \%$ & 0.540 & 0.834 & 0.628 & 0.499 & 0.650 & 0.730 & 0.670 & 0.92 & 0.130 \\
\hline Relative importance of gca \% ${ }^{*}$ & 55.390 & 84.660 & 71.300 & 52.230 & 72.110 & 79.300 & 70.000 & 98.530 & 13.260 \\
\hline Relative importance of sca \%** & 44.610 & 15.340 & 28.700 & 47.770 & 27.890 & 20.700 & 30.010 & 1.410 & 86.750 \\
\hline
\end{tabular}

Relative importance of sca \%**

\begin{tabular}{l|l|l}
44.610 & 15.340 & 28.700 \\
\hline
\end{tabular}

${ }^{*},{ }^{\star \star}$ Relative importance gca $=\sigma^{2} A / \sigma^{2} G \times 100$ and Relative importance sca $=\sigma^{2} D / \sigma^{2} G \times 100$, respectively.

Table (4): General combining ability effects of CMS lines (A lines).

\begin{tabular}{|c|c|c|c|c|c|c|c|c|c|}
\hline Lines & $\begin{array}{l}\text { Heading } \\
\text { date } \\
\text { (days) }\end{array}$ & $\begin{array}{l}\text { Plant height } \\
\text { (cm) }\end{array}$ & $\begin{array}{c}\text { Panicle } \\
\text { length }(\mathrm{cm})\end{array}$ & $\begin{array}{c}\text { Panicle } \\
\text { weight(g) }\end{array}$ & $\begin{array}{c}\text { Filled grains } \\
\text { panicle }\end{array}$ & $\begin{array}{c}\text { Spikelets } \\
\text { panicle }\end{array}$ & $\begin{array}{c}\text { Spikelet } \\
\text { fertility \% }\end{array}$ & $\begin{array}{c}\text { 1000-grain } \\
\text { weight(g) }\end{array}$ & $\begin{array}{c}\text { Grain yield } \\
\mathrm{t} / \mathrm{ha}\end{array}$ \\
\hline IR68885A & $-2.9167^{\star *}$ & $-1.1667^{* *}$ & $0.6611^{* *}$ & $-0.7428^{\star *}$ & $-25.1181^{* *}$ & $-27.2333^{\star *}$ & $-1.5972^{* *}$ & $-2.6806^{* *}$ & $-0.9267^{* *}$ \\
\hline IR69625A & $-1.4722^{* *}$ & $-6.8333^{* *}$ & 0.1722 & $0.3717^{* *}$ & 1.8419 & $-8.3000^{* *}$ & $5.4361^{\star *}$ & $-0.5028^{*}$ & $0.9744^{* *}$ \\
\hline G46A & $2.8611^{* *}$ & $7.2778^{* *}$ & -0.1167 & $0.5039^{* *}$ & $11.2075^{\star *}$ & $15.5556^{* *}$ & $-1.1306^{* *}$ & $1.3417^{* *}$ & $0.5422^{* *}$ \\
\hline Large stigmaA & $1.5278^{* *}$ & $0.7222^{*}$ & $-.7167^{\star *}$ & $-0.1328^{\star *}$ & $12.0686^{* *}$ & $19.9778^{* *}$ & $-2.7083^{* *}$ & $1.8417^{* *}$ & $-0.5900^{\star *}$ \\
\hline LSD 5\% & 0.66 & 0.721 & 0.24 & 0.108 & 4.37 & 4.69 & .0673 & 0.401 & 0.15 \\
\hline $1 \%$ & 0.88 & 0.964 & 0.321 & 0.145 & 5.84 & 6.27 & 1.02 & 0.536 & 0.199 \\
\hline
\end{tabular}


* , ** Significant at 0.05 and 0.01 levels, respectively.

Table (5): General combining ability effects of testers.

\begin{tabular}{|c|c|c|c|c|c|c|c|c|c|}
\hline Tester & $\begin{array}{c}\text { Heading date } \\
\text { (days) }\end{array}$ & $\begin{array}{c}\text { Plant height } \\
\text { (cm) }\end{array}$ & $\begin{array}{l}\text { Panicle } \\
\text { length } \\
(\mathrm{cm})\end{array}$ & $\begin{array}{l}\text { Panicle } \\
\text { weight } \\
\text { (g) }\end{array}$ & $\begin{array}{c}\text { Filled grains } \\
\text { panicle }^{-1}\end{array}$ & $\begin{array}{l}\text { Spikelets } \\
\text { panicle }^{-1}\end{array}$ & $\begin{array}{c}\text { Spikelet } \\
\text { fertility } \\
(\%)\end{array}$ & $\begin{array}{c}\text { 1000-grain } \\
\text { weight(g) }\end{array}$ & $\begin{array}{l}\text { Grain } \\
\text { Yield } \\
\text { (t/ha) }\end{array}$ \\
\hline $\begin{array}{l}\text { Giza 178R } \\
\text { Giza182R } \\
\text { GZ 5121R }\end{array}$ & $\begin{array}{c}-2.7778^{\star \star} \\
-3.1944^{\star *} \\
5.9722^{\star *}\end{array}$ & $\begin{array}{l}-4.5556^{\star \star} \\
-2.2222^{\star \star} \\
6.7778^{\star \star}\end{array}$ & $\begin{array}{c}0.02780 \\
0.71110^{\star *} \\
-0.7389^{\star *}\end{array}$ & $\begin{array}{c}0.1975^{\star \star} \\
0.2250^{\star \star} \\
-0.4225^{\star \star}\end{array}$ & $\begin{array}{c}5.8856^{\star \star} \\
4.2739^{\star} \\
-10.1594^{\star \star}\end{array}$ & $\begin{array}{c}3.1389 \\
1.4472 \\
-4.5861^{*}\end{array}$ & $\begin{array}{c}1.5167^{* \star} \\
2.0250^{\star *} \\
-3.5417^{\star *}\end{array}$ & $\begin{array}{c}-0.8778^{\star \star} \\
0.4139^{\star} \\
0.4639^{\star *}\end{array}$ & $\begin{array}{l}0.7489^{* \star} \\
-0.0903^{\star *} \\
-0.6586^{\star *}\end{array}$ \\
\hline$\frac{1 \%}{1 \%}$ & $\begin{array}{l}0.5702 \\
0.7600\end{array}$ & $\begin{array}{l}0.1110 \\
0.6240 \\
0.8350\end{array}$ & $\begin{array}{r}-1005 \\
0.2080 \\
0.2780\end{array}$ & $\begin{array}{l}0.4<25 \\
0.0940 \\
0.1250\end{array}$ & $\begin{array}{l}3.1800 \\
5.0600\end{array}$ & $\begin{array}{r}-4.0601 \\
5.4300\end{array}$ & $\begin{array}{l}0.0411 \\
0.6610 \\
0.8840\end{array}$ & $\begin{array}{l}0.4005 \\
0.3470 \\
0.4640\end{array}$ & $\begin{array}{l}-0.0500 \\
0.1300 \\
0.1700\end{array}$ \\
\hline
\end{tabular}

** Significant at 0.05 and 0.01 levels, respectively.

Table (6): Specific combining ability of hybrid combinations.

\begin{tabular}{|c|c|c|c|c|c|c|c|c|c|}
\hline Hybrid combination & $\begin{array}{l}\text { Heading } \\
\text { date } \\
\text { (days) }\end{array}$ & $\begin{array}{l}\text { Plant } \\
\text { height } \\
\text { (cm) }\end{array}$ & $\begin{array}{l}\text { Panicle } \\
\text { length } \\
\text { (cm) }\end{array}$ & $\begin{array}{l}\text { Panicle } \\
\text { weight } \\
\text { (g) }\end{array}$ & $\begin{array}{l}\text { Filled grains } \\
\text { panicle }^{-1}\end{array}$ & $\begin{array}{l}\text { Spikelets } \\
\text { panicle }^{-1}\end{array}$ & $\begin{array}{c}\text { Spikelet } \\
\text { fertility } \\
(\%)\end{array}$ & $\begin{array}{l}\text { 1000-grain } \\
\text { weight(g) }\end{array}$ & $\begin{array}{l}\text { Grain } \\
\text { yield } \\
\text { (t/ha) }\end{array}$ \\
\hline IR68885A /Giza178R & $-1.667^{\star \star}$ & $-1.333^{*}$ & -0.261 & $-0.610^{* *}$ & $-8.49778^{*}$ & $\begin{array}{l}-3.55000 \\
-525832\end{array}$ & $-3.06111^{* *}$ & 0.12222 & -0.15000 \\
\hline $\begin{array}{l}\text { /Giza182R } \\
\text { /GZ 5121R }\end{array}$ & $\begin{array}{l}2.750^{\star *} \\
-1.083\end{array}$ & $\begin{array}{l}-1.333^{*} \\
2.667^{\star *}\end{array}$ & $\begin{array}{l}0.189 \\
0.072\end{array}$ & $\begin{array}{l}0.403^{\star \star *} \\
0.207^{\star}\end{array}$ & $\begin{array}{l}-6.51944 \\
15.01722^{* *}\end{array}$ & $\begin{array}{l}-5.25833 \\
8.80833^{*}\end{array}$ & $\begin{array}{l}-1.40278^{*} \\
4.46389^{\star *}\end{array}$ & $\begin{array}{l}-0.00278 \\
-0.11944\end{array}$ & $\begin{array}{l}-1.55417^{\star *} \\
1.70417^{\star \star}\end{array}$ \\
\hline IR69625A /Giza178R & $3.556^{\star *}$ & -0.333 & -0.339 & $-0.218^{\star}$ & $11.54222^{\star \star}$ & $13.71667^{\star \star}$ & -0.42778 & 0.17778 & $-0.33444^{*}$ \\
\hline Giza182R & $2.639^{* *}$ & $-3.333^{\star \star}$ & $0.478^{*}$ & $-0.228^{*}$ & $-8.17611^{\star}$ & $-12.05833^{* *}$ & $2.03056^{* *}$ & & -0.00861 \\
\hline $\begin{array}{l}\text { / GZ 5121R } \\
\text { G46A/ Giza 78R }\end{array}$ & $\begin{array}{l}-6.194^{* *} \\
-1.111\end{array}$ & $3.667^{* *}$ & $\begin{array}{l}-0.139 \\
0.183\end{array}$ & $0.446^{\star *}$ & $\begin{array}{l}-3.36611 \\
-5\end{array}$ & $\begin{array}{r}-1.65 \\
-1017\end{array}$ & $\begin{array}{l}-1.60278^{*} \\
19722^{* *}\end{array}$ & 0.136 & $\begin{array}{l}0.34306^{*} \\
0.23444\end{array}$ \\
\hline Giza182R & & $2.556^{* *}$ & $-0.767^{\star \star}$ & 0.053 & 7.6350 & $10.08611^{*}$ & 6944 & 0.508 & $1.44694^{\star *}$ \\
\hline GZ 5121R & $3.472^{* *}$ & $-4.111^{\star *}$ & $0.583^{* *}$ & $-0.593^{\star *}$ & -2.24500 & & -1.10278 & 0.15 & $-1.68139^{\star *}$ \\
\hline Large stigma A /Giza178R & -0.778 & 0.111 & $0.417^{\star}$ & $0.287^{\text {** }}$ & 2.34556 & 0.005 & $1.51667^{\star *}$ & 0.36667 & 0.25000 \\
\hline $\begin{array}{l}\text { Giza182R } \\
\text { / GZ 5121R }\end{array}$ & $\begin{array}{l}-3.028^{* *} \\
3.806^{* *}\end{array}$ & $\begin{array}{l}2.111^{\star *} \\
-2.222^{* *}\end{array}$ & $\begin{array}{c}0.100 \\
-0.517^{\star}\end{array}$ & $\begin{array}{l}-0.227^{*} \\
-0.060\end{array}$ & $\begin{array}{l}7.06056 \\
-9.40611^{\star}\end{array}$ & $\begin{array}{r}7.23056 \\
-7.23611\end{array}$ & $\begin{array}{c}0.24167 \\
-1.75833^{\star}\end{array}$ & $\begin{array}{l}-0.19167 \\
-0.17500\end{array}$ & $\begin{array}{c}0.11583 \\
-0.36583^{* *}\end{array}$ \\
\hline LSD 5\% & 1.14300 & & 0.41600 & 0.18700 & 7.56000 & 8.12000 & 1.32000 & 0.69400 & 0.26000 \\
\hline $1 \%$ & 1.53000 & 1.67000 & 0.55700 & 0.25000 & 10.12000 & 10.86000 & 1.77000 & 0.92800 & 0.35000 \\
\hline
\end{tabular}

${ }^{*},{ }^{*}$ Significant at 0.05 and 0.01 levels, respectively.

Table (7): Estimates of heterosis over better-parent (BP) for 9 characters of 12 hybrid combinations.

\begin{tabular}{|c|c|c|c|c|c|c|c|c|c|}
\hline \multirow{2}{*}{\begin{tabular}{|c|} 
Hybrid combination \\
R68885A / Giza178R
\end{tabular}} & \multirow{2}{*}{$\begin{array}{c}\begin{array}{c}\text { Heading } \\
\text { date } \\
\text { (days) }\end{array} \\
-9.00^{* *} \\
\end{array}$} & \multirow[b]{2}{*}{$\begin{array}{l}\text { Plant } \\
\text { height } \\
(\mathrm{cm})\end{array}$} & \multirow[b]{2}{*}{$\begin{array}{l}\text { Panicle } \\
\text { length } \\
\text { (cm) }\end{array}$} & \multirow[b]{2}{*}{$\begin{array}{l}\text { Panicle } \\
\text { weight } \\
\text { (g) }\end{array}$} & \multirow[b]{2}{*}{\begin{tabular}{|} 
Filled grains \\
panicle $^{-1}$
\end{tabular} \mid} & \multirow[b]{2}{*}{$\begin{array}{l}\text { Spikelets } \\
\text { panicle }^{-1}\end{array}$} & \multirow[b]{2}{*}{$\begin{array}{l}\text { Spikelet } \\
\text { fertility } \\
(\%)\end{array}$} & \multirow[b]{2}{*}{$\begin{array}{l}\text { 1000-grain } \\
\text { weight(g) }\end{array}$} & \multirow[b]{2}{*}{$\begin{array}{l}\text { Grain } \\
\text { yield } \\
\text { (t/ha) } \\
975^{* *}\end{array}$} \\
\hline & & & & & & & & & \\
\hline \begin{tabular}{|cc} 
IR68885A & / Giza178R \\
& /Giza182R \\
& /GZ5121R
\end{tabular} & $\begin{array}{l}-9.00^{\star *} \\
1.33 \\
-2.20^{\star \star}\end{array}$ & $\begin{array}{l}-0.39 \\
9.30^{* *} \\
15.60^{\star *}\end{array}$ & $\begin{array}{l}7.90^{* *} \\
13.10^{* *} \\
13.80^{* *}\end{array}$ & $\begin{array}{l}-2.70 \\
24.30^{\star *} \\
-9.40^{*}\end{array}$ & \begin{tabular}{|l|}
$-12.80^{* *}$ \\
$-16.40^{\star *}$ \\
-6.10
\end{tabular} & $\begin{array}{l}-12.20^{* *} \\
-10.40^{\star \star} \\
2.03\end{array}$ & $\begin{array}{c}0.73 \\
-6.60^{\star *} \\
-8.00^{\star *}\end{array}$ & $\begin{array}{l}8.04 \\
3.70 \\
3.66\end{array}$ & $\begin{array}{r}.75^{\star *} \\
-12.24^{\star \star} \\
12.22^{\star \star}\end{array}$ \\
\hline $\begin{array}{l}\text { / Giza178R } \\
\text { / Giza182R }\end{array}$ & $\begin{array}{l}-2.60^{\star *} \\
-2.80^{\star *}\end{array}$ & $\begin{array}{c}-4.80^{\star *} \\
1.30^{\star *}\end{array}$ & $\begin{array}{c}5.50^{* *} \\
12.20^{* *}\end{array}$ & $\begin{array}{l}36.50^{* *} \\
37.00^{* *}\end{array}$ & $\begin{array}{l}17.90^{* *} \\
1.50\end{array}$ & $\begin{array}{l}9.60^{\star *} \\
-2.80\end{array}$ & $\begin{array}{l}11.10^{* *} \\
5.20^{\star *}\end{array}$ & $\begin{array}{c}22.30 \\
3.30\end{array}$ & $\begin{array}{l}26.60^{* *} \\
21.82^{* *}\end{array}$ \\
\hline / GZ5121R & $-5.60^{* *}$ & $11.00^{\star *}$ & $10.70^{* *}$ & $23.30^{* *}$ & 0.30 & $7.80^{*}$ & $-6.90^{* *}$ & 5.10 & $20.60^{* *}$ \\
\hline $\begin{array}{l}\text { / Giza 178R } \\
\text { /Giza182R }\end{array}$ & $-2.90^{* *}$ & & $6.70^{\star *}$ & $60.50^{* *}$ & $16.20^{\star *}$ & $9.61^{* *}$ & $6.00^{\star *}$ & & $27.98^{\star \star}$ \\
\hline $\begin{array}{r}\text { /Giza182R } \\
\text { / GZ5121R }\end{array}$ & $\begin{array}{l}2.00^{* *} \\
750^{* *}\end{array}$ & $\begin{array}{l}22.00^{* *} \\
1710^{* *}\end{array}$ & $\begin{array}{l}5.60^{\star \star} \\
1250^{\star *}\end{array}$ & $\begin{array}{c}48.10^{\star \star \star} \\
1.50\end{array}$ & $\begin{array}{l}19.20^{* *} \\
8.10^{* *}\end{array}$ & $\begin{array}{l}26.14^{\star \star} \\
25.15^{\star \star}\end{array}$ & $\begin{array}{l}-5.50^{\star *} \\
-13.60^{* *}\end{array}$ & $\begin{array}{c}13.2 \\
1210\end{array}$ & $\begin{array}{l}31.89 \\
-378^{* *}\end{array}$ \\
\hline Large stigma A / Giza178R & $-3.8^{\star *}$ & $2.90^{\star \star}$ & $5.00^{* *}$ & $36.50^{* *}$ & $22.50^{* *}$ & $18.40^{* *}$ & $3.60^{\star *}$ & $33.50^{*}$ & $16.95^{\star *}$ \\
\hline$/ G Z 5121 R$ & $\begin{array}{l}0.00 \\
6.50 \text { ** }\end{array}$ & $\begin{array}{l}14.80^{\star *} \\
12.70^{\star *}\end{array}$ & $\begin{array}{l}6.80^{\star *} \\
4.90^{\star *}\end{array}$ & $\begin{array}{l}23.20^{\star *} \\
-1.20\end{array}$ & $\begin{array}{l}19.50^{\star *} \\
3.40\end{array}$ & $\begin{array}{l}27.15^{\star \star} \\
23.20^{\star \star}\end{array}$ & $\begin{array}{l}-6.10^{* *} \\
-16.10^{* *}\end{array}$ & $\begin{array}{l}12.50 \\
12.50\end{array}$ & $\begin{array}{l}7.60^{\star \star} \\
-1.99\end{array}$ \\
\hline
\end{tabular}




\begin{tabular}{|l|l|l|l|l|l|l|l|l|l|}
\hline LSD 5\% & 1.62 & 1.98 & 0.59 & 0.27 & 10.69 & 11.48 & 1.87 & 5.89 & 0.37 \\
\hline $1 \%$ & 2.16 & 2.65 & 0.79 & 0.36 & 14.31 & 15.36 & 2.5 & 7.88 & 0.49 \\
\hline
\end{tabular}

${ }^{*},{ }^{\star *}$ Significant at 0.05 and 0.01 levels, respectively.

Table (8): Estimates of heterosis mid-parents (MP) for 9 characters of 12 hybrid combinations.

\begin{tabular}{|c|c|c|c|c|c|c|c|c|c|}
\hline Hybrid combination & $\begin{array}{l}\text { Heading } \\
\text { date } \\
\text { (days) }\end{array}$ & $\begin{array}{l}\text { Plant } \\
\text { height } \\
(\mathrm{cm})\end{array}$ & $\begin{array}{l}\text { Panicle } \\
\text { length } \\
\text { (cm) }\end{array}$ & $\begin{array}{l}\text { Panicle } \\
\text { weight } \\
\text { (g) }\end{array}$ & $\begin{array}{l}\text { Filled grains } \\
\text { panicle }^{-1}\end{array}$ & $\begin{array}{l}\text { Spikelets } \\
\text { panicle }^{-1}\end{array}$ & $\begin{array}{c}\text { Spikelet } \\
\text { fertility } \\
(\%)\end{array}$ & $\begin{array}{l}1000 \text {-grain } \\
\text { weight(g) }\end{array}$ & $\begin{array}{c}\text { Grain yield } \\
(\mathrm{t} / \mathrm{ha})\end{array}$ \\
\hline / Giza178R & $-7.40^{* \star}$ & $3.60^{* \star}$ & $5.70^{* \star}$ & 5.60 & -6.90 & -5.20 & -2.00 & 8.30 & $17.88^{\star \star}$ \\
\hline /Giza182R & -0.20 & $9.90^{* *}$ & $15.30^{* *}$ & $35.30^{* *}$ & $-9.70^{*}$ & -5.30 & $-3.60^{* *}$ & 6.10 & $-5.83^{* *}$ \\
\hline /GZ5121R & 0.67 & $19.70^{* *}$ & $12.30^{* *}$ & 3.90 & -0.40 & 4.10 & $-4.20^{* *}$ & 6.05 & $23.19^{\star *}$ \\
\hline / Giza178R & $-3.30^{\star *}$ & $-6.60^{* *}$ & $5.00^{* *}$ & $29.50^{* *}$ & $23.30^{* *}$ & $18.10^{* *}$ & $3.80^{* \star}$ & 10.30 & $29.41^{* *}$ \\
\hline / Giza182R & $-1.50^{\star}$ & $-4.10^{* *}$ & $11.20^{* *}$ & $30.00^{* *}$ & 5.00 & 2.70 & $2.90^{\star *}$ & 3.30 & $24.40^{\star *}$ \\
\hline / GZ5121R & $5.30^{* *}$ & $8.40^{\star *}$ & $6.00^{* \star}$ & $24.20^{\star *}$ & 0.83 & $9.80^{* *}$ & $-8.14^{\star *}$ & 5.10 & $22.67^{\star *}$ \\
\hline G46A / Giza 178R & $-4.70^{\star *}$ & $11.10^{\star *}$ & $10.30^{* *}$ & $44.90^{* *}$ & $14.20^{* *}$ & $10.10^{\star *}$ & $3.60^{\star *}$ & 13.00 & $41.81^{* *}$ \\
\hline /Giza182R & $-3.30^{\star *}$ & $18.40^{\star *}$ & $9.10^{* \star}$ & $33.70^{* *}$ & $19.80^{* *}$ & $23.98^{\star \star}$ & $-3.40^{\star *}$ & 12.20 & $46.00^{\star *}$ \\
\hline / GZ5121R & $6.60^{* *}$ & $17.10^{* *}$ & $12.50^{\star *}$ & -2.60 & & $18.30^{* *}$ & $-11.00^{* *}$ & & $6.15^{\star \star}$ \\
\hline Large stigma A / Giza178R & $7.70^{* *}$ & $10.00^{\star *}$ & $14.40^{* *}$ & $48.50^{* *}$ & $41.90^{* *}$ & $44.53^{\star *}$ & $-3.30^{* *}$ & 12.80 & $31.89^{\star *}$ \\
\hline & $8.60^{* *}$ & $18.70^{* *}$ & $16.40^{* *}$ & $34.10^{* *}$ & $40.80^{* *}$ & $52.60^{* *}$ & $-8.10^{* *}$ & 6.10 & $21.25^{\star *}$ \\
\hline / GZ5121R & $20.40^{\star \star}$ & $19.90^{\star \star}$ & $10.90^{\star *}$ & $13.30^{\star *}$ & $19.10^{* *}$ & $43.40^{\star \star}$ & $-17.20^{\star \star}$ & 6.1 & $10.06^{\star *}$ \\
\hline LSD 5\% & 1.40 & 1.72 & 0.51 & 0.23 & 9.26 & $9.94 \quad 13.30$ & 1.62 & 5.10 & 0.32 \\
\hline $1 \%$ & 1.87 & 2.29 & 0.68 & 0.31 & 12.3 & & 2.17 & 6.82 & 0.42 \\
\hline
\end{tabular}

${ }^{\star}{ }^{* \star}$ Significant at 0.05 and 0.01 levels, respectively.

\section{Table (9): Standard heterosis for 9 characters of 12 hybrid combinations.}

\begin{tabular}{|c|c|c|c|c|c|c|c|c|c|}
\hline Hybrid combination & $\begin{array}{l}\text { Heading } \\
\text { date } \\
\text { (days) }\end{array}$ & $\begin{array}{c}\text { Plant height } \\
\text { (cm) }\end{array}$ & $\begin{array}{l}\text { Panicle } \\
\text { length } \\
(\mathrm{cm})\end{array}$ & $\begin{array}{l}\text { Panicle } \\
\text { weight } \\
\text { (g) }\end{array}$ & $\begin{array}{c}\text { Filled grains } \\
\text { panicle }^{-1}\end{array}$ & $\begin{array}{l}\text { Spikelets } \\
\text { panicle }^{-1}\end{array}$ & $\begin{array}{c}\text { Spikelet } \\
\text { fertility (\%) })\end{array}$ & $\begin{array}{c}\text { 1000-grain } \\
\text { weight(g) }\end{array}$ & $\begin{array}{c}\text { Grain yield } \\
\text { (t/ha) }\end{array}$ \\
\hline / Giza178R & $-9.00^{* *}$ & -0.39 & $7.90^{* \star}$ & -2.70 & $-12.80^{\star \star}$ & $-12.20^{* *}$ & -0.73 & 8.04 & $9.75^{* *}$ \\
\hline /Giza182R & $-5.20^{* *}$ & $1.93^{\star \star}$ & $12.90^{\star *}$ & $24.30^{* *}$ & $-14.22^{* *}$ & $-14.22^{* *}$ & 2.00 & 17.40 & $-12.41^{\star *}$ \\
\hline /GZ5121R & -2.20 & $14.50^{\star \star}$ & $6.30^{\star \star}$ & 1.10 & -8.00 & $-9.41^{\star *}$ & $2.30^{*}$ & 17.40 & $14.10^{\star \star}$ \\
\hline / Giza178R & $-2.60^{\star *}$ & $-4.80^{\star \star}$ & $5.50^{\star \star}$ & $36.50^{\star *}$ & $17.90^{\star *}$ & $9.60^{\star \star}$ & $11.10^{\star \star}$ & 22.30 & $26.60^{* *}$ \\
\hline / Giza182R & $-3.80^{* *}$ & $-5.50^{\star *}$ & $12.10^{* *}$ & $37.00^{* *}$ & 6.04 & $-6.94^{*}$ & $14.80^{* *}$ & 25.90 & $21.58^{\star \star}$ \\
\hline / GZ5121R & $-3.50^{* *}$ & $9.90^{* *}$ & $3.30^{* *}$ & $37.60^{* *}$ & -1.03 & -4.29 & $3.60^{* *}$ & $28.10^{\star *}$ & $19.41^{* *}$ \\
\hline / Giza 178R & $-2.90^{* *}$ & $10.61^{* *}$ & $6.70^{\star \star *}$ & $60.50^{* *}$ & $16.20^{\star *}$ & $9.60^{* *}$ & $6.00^{* *}$ & $26.80^{*}$ & $27.98^{\star \star}$ \\
\hline /Giza182R & $-4.50^{\star}$ & $13.80^{\star *}$ & $5.40^{\star *}$ & $48.10^{* *}$ & $24.60^{* *}$ & $20.80^{* *}$ & $3.20^{\star \star}$ & $38.00^{* *}$ & $31.63^{\star *}$ \\
\hline / GZ5121R & $9.80^{* *}$ & $16.00^{\star *}$ & $5.00^{\star *}$ & $13.20^{* *}$ & 6.70 & $11.12^{* \star}$ & $-3.90^{\star *}$ & $36.60^{\star *}$ & $-4.73^{\star \star}$ \\
\hline Large stigma A / Giza178R & $-3.80^{\star *}$ & $2.90^{\star \star}$ & $5.00^{\star *}$ & $36.50^{\star *}$ & $22.50^{\star *}$ & $18.40^{\star \star}$ & $3.60^{\star *}$ & $33.50^{\star \star}$ & $16.95^{\star \star}$ \\
\hline & $-6.40^{* *}$ & $7.04^{\star *}$ & $6.70^{\star \star}$ & $23.20^{* *}$ & $24.80^{\star *}$ & $21.70^{\star *}$ & $2.60^{* *}$ & $37.10^{\star \star}$ & $7.39^{* *}$ \\
\hline / GZ5121R & $8.90^{* *}$ & $11.60^{* *}$ & -2.10 & $10.30^{* *}$ & 2.10 & $8.57^{* *}$ & $-6.60^{* *}$ & $37.10^{\star *}$ & -2.96 \\
\hline LSD 5\% & 1.62 & 1.98 & 0.59 & 0.27 & 10.69 & 11.48 & 1.87 & 5.89 & 0.37 \\
\hline $1 \%$ & 2.16 & 2.65 & 0.79 & 0.36 & 14.31 & 15.36 & 2.5 & 7.88 & 0.49 \\
\hline
\end{tabular}

${ }^{\star},{ }^{* \star}$ Significant at 0.05 and 0.01 levels, respectively. 